\title{
New $\gamma$ Cassiopeiae-like objects: X-ray and optical observations of SAO 49725 and HD 161103^
}

\author{
R. Lopes de Oliveira ${ }^{1,2}$, C. Motch ${ }^{1}$, F. Haberl ${ }^{3}$, I. Negueruela ${ }^{4}$, and E. Janot-Pacheco ${ }^{2}$ \\ 1 Observatoire Astronomique, UMR 7550 CNRS, 11 rue de l'Université, 67000 Strasbourg, France \\ e-mail: rlopes@newb6.u-strasbg.fr \\ 2 Instituto de Astronomia, Geofísica e Ciências Atmosféricas, Universidade de São Paulo, R. do Matão 1226, \\ 05508-090 São Paulo, Brazil \\ 3 Max-Planck-Institut für extraterrestrische Physik, Giessenbachstraße, 85748 Garching, Germany \\ ${ }^{4}$ Departamento de Física, Ingeniería de Sistemas y Teoría de la Señal, Escuela Politécnica Superior, Universidad de Alicante, \\ Ap. 99, 03080 Alicante, Spain
}

Received 25 November 2005 / Accepted 28 February 2006

\begin{abstract}
A growing number of early Be stars exhibit X-ray luminosities that are intermediate between those typical of early type stars and those emitted by most Be/X-ray binaries in quiescence. We report on XMM-Newton observations of two such Be stars, SAO 49725 and HD 161103, which were originally discovered in a systematic cross-correlation between the ROSAT all-sky survey and SIMBAD. The new observations confirm the X-ray luminosity excess $\left(L_{\mathrm{X}} \sim 10^{32-33} \mathrm{erg} \mathrm{s}^{-1}\right)$ and the hardness of their X-ray spectra, which are both unusual for normal early type stars. An iron K $\alpha$ complex is clearly detected in HD 161103 in which the H-like, He-like, and fluorescent components are resolved, while strong evidences also exist for the presence of similar features in SAO 49725. X-ray spectra can be equally well-fitted by a thermal plasma (mekal) with $T \sim 10^{8} \mathrm{~K}$ and solar abundances or by a power law + iron line model with photon index $\sim 1.5-1.8$, both with a soft thermal component with $T \sim 10^{7} \mathrm{~K}$. The intensity of the fluorescence $6.4 \mathrm{keV}$ line reflects the presence of large amounts of cold material close to the X-ray sources and strongly argues against accretion onto a companion neutron star in a large orbit. On the other hand, the probable thermal origin of the X-ray emission, as supported by the ionised iron lines, disagrees with those observed in all known Be/X-ray binaries, in which a non-thermal component is always required. Remarkably, the X-ray features are similar to those of white dwarves in several cataclysmic variables. There is no evidence of high frequency pulsations in neither of the two systems. However, a large oscillation in the light curve of HD 161103 with $P \sim$ $3200 \mathrm{~s}$ is readily detected. The X-ray light curve of SAO 49725 exhibits clear variability by $\sim 80 \%$ on time scales as short as $\sim 1000 \mathrm{~s}$. New optical observations provide updated spectral types (B0.5 III-Ve) and disclose a dense, large, and apparently stable circumstellar disc for both stars. The nature of the excess X-ray emission is discussed in light of the models proposed for $\gamma$ Cas, magnetic disc-star interaction, or accretion onto a compact companion object - whether neutron star or white dwarf. These two new objects, added to similar cases discovered in XMM-Newton surveys, point to the emergence of a new class of $\gamma$ Cas analogs.
\end{abstract}

Key words. stars: emission-line, Be - stars: individual: SAO 49725- stars: individual: HD 161103

\section{Introduction}

SAO 49725 and HD 161103 are two B0.5 III-Ve stars identified as relatively strong $\mathrm{X}$-ray emitters through cross-correlation between the ROSAT all-sky survey and O-B star catalogs (Motch et al. 1997). The short ROSAT observation did not allow detailed spectral studies but did show that the two sources had harder X-ray spectra than those of normal O-B stars and luminosities $\left(\sim 10^{32} \mathrm{erg} \mathrm{s}^{-1}\right.$ in the $0.1-2.4 \mathrm{keV}$ energy range) midway between those radiated by normal stars and classical Be/X-ray transients in quiescence, as well as persistent low-luminosity Be/X-ray binaries (X Per-like objects). Based on these peculiarities, Motch et al. (1997) suggested that SAO 49725 and HD 161103 were very likely new $\mathrm{Be} / \mathrm{X}$-ray binaries and possible $\mathrm{Be}+$ white dwarf (Be+WD) systems, owing to the modest $\mathrm{X}$-ray luminosity.

The Be/X-ray binaries constitute the most numerous class of high-mass X-ray binaries ( $2 / 3$ of them) in which gravitational

* Based on observations obtained with XMM-Newton, an ESA science mission with instruments and contributions directly funded by ESA Member States and NASA. capture onto a compact object of the Be star's circumstellar material accounts for the X-ray emission (see Raguzova \& Popov 2005, for a recent compilation of Be/X-ray properties). The $\mathrm{Be}+\mathrm{WD}$ systems are produced in large numbers by models describing the evolution of massive binaries, in which $20 \%$ to $70 \%$ of the Be stars formed as the result of the binary evolution must have a white dwarf as a companion (see e.g. van den Heuvel \& Rappaport 1987; Waters et al. 1989; Pols et al. 1991; van Bever \& Vanbeveren 1997; Raguzova 2001). In spite of their high expected frequency, no such $\mathrm{Be}+\mathrm{WD}$ binary has been firmly established yet. The only tenable $\mathrm{Be}+\mathrm{WD}$ candidate known so far is $\gamma$ Cas, whose X-ray and optical properties are similar to those of SAO 49725 and HD 161103. In almost all well-studied $\mathrm{Be} / \mathrm{X}$-ray binaries, the X-ray spectra, the peak luminosities, pulsations, spin up rates, and magnetic fields altogether or separately point to a neutron star (NS).

In this paper we present the spectral and timing analysis of new X-ray observations of SAO 49725 and HD 161103 obtained by the XMM-Newton satellite and optical spectroscopy carried out during several observational campaigns. The X-ray and 
Table 1. Journal of optical spectroscopic observations.

\begin{tabular}{cccc}
\hline \hline Date & Telescope & $\begin{array}{c}\text { Wavelength } \\
\text { range }(\AA)\end{array}$ & $\begin{array}{c}\text { Dispersion } \\
(\AA / \text { pixel })\end{array}$ \\
\hline \multicolumn{4}{c}{ SAO 49725 } \\
$28 / 09 / 95$ & OHP 193 & $3750-6750$ & 6.9 \\
$18 / 08 / 99$ & Cassini & $4100-6700$ & 1.9 \\
$25 / 07 / 00$ & Cassini & $3100-5300$ & 1.6 \\
$25 / 07 / 00$ & Cassini & $6150-8150$ & 1.6 \\
$31 / 07 / 01$ & OHP 152 & $3960-4410$ & 0.22 \\
$01 / 08 / 01$ & OHP 152 & $4475-4900$ & 0.22 \\
$02 / 08 / 01$ & OHP 152 & $6380-6830$ & 0.22 \\
$22 / 10 / 01$ & OHP 193 & $6250-7120$ & 0.45 \\
$24 / 10 / 01$ & OHP 193 & $3800-5500$ & 0.90 \\
$01 / 07 / 03$ & INT & $5300-7000$ & 0.65 \\
$02 / 07 / 03$ & INT & $3800-5500$ & 0.65 \\
$17 / 07 / 04$ & Cassini & $3900-10200^{a}$ & 0.8 \\
$06 / 07 / 05$ & Cassini & $3900-10200^{a}$ & 0.8 \\
\hline \multicolumn{4}{c}{ HD 161103 } \\
$13 / 02 / 94$ & ESO 1.5 m & $4050-7200$ & 1.9 \\
$01 / 08 / 98$ & INT & $3670-5060$ & 0.5 \\
$02 / 08 / 98$ & INT & $5800-7100$ & 0.5 \\
$03 / 08 / 98$ & INT & $7700-8950$ & 0.5 \\
$01 \ldots 05 / 03 / 03^{b}$ & ESO 3.6 m & $3600-5050$ & 1.9 \\
$07 / 06 / 03$ & NTT & $3980-4765$ & 0.8 \\
$07 / 06 / 03$ & NTT & $6320-7830$ & 0.4 \\
\hline \multicolumn{4}{c}{}
\end{tabular}

${ }^{a}$ Covered in different orders; ${ }^{b}$ five observations (one per night).

optical properties of SAO 49725 and HD 161103 are notably close to those of $\gamma$ Cas. We discuss the nature of the X-ray emission in both objects in the light of the single-star and binary models proposed for $\gamma$ Cas.

\section{Observations}

\subsection{Optical observations}

Optical spectroscopy of SAO 49725 and HD 161103 was obtained with a variety of telescopes and instruments, listed in Table 1.

SAO 49725 was observed at the Observatoire de Haute Provence (OHP, France) with the 1.93-m telescope and the Carelec spectrograph on 28 September 1995, and on 22 and 24 October 2001. Other observations were taken at OHP with the 1.52-m telescope and the Elodie spectrograph between 31 July and 2 August 2001. Four observing runs were performed with the 1.52-m G. D. Cassini telescope at the Loiano Observatory (Bologna, Italy) on 18 August 1999, 25 July 2000, 17 July 2004, and in 6 July 2005. On all occasions, the telescope was equipped with the Bologna faint-object spectrograph and camera (BFOSC). Several grisms were used, in order to cover both the blue and $\mathrm{H} \alpha$ regions at moderate resolutions $(\sim 3-4 \AA)$. Finally, data were taken with the 2.5-m Isaac Newton Telescope (INT) at La Palma (Spain) and the intermediate dispersion spectrograph (IDS) on 1 and 2 July 2003. Details of the configurations are shown in Table 1.

HD 161103 was observed in low dispersion with a Boller $\mathcal{F}$ Chivens spectrograph mounted at the 1.5-m ESO on 13 February 1994. Medium resolution spectra were acquired with the INT + IDS on 1-3 August 1998 (data retrieved from the archive). Observations were carried out during five consecutive nights in March 2003 using the ESO 3.6-m telescope equipped with the EFOSC instrument, with the ESO-NTT at La Silla, and the ESO multi-mode instrument (EMMI) on 7 June 2003.

\subsection{X-ray observations}

XMM-Newton observed SAO 49725 on 9 December 2003 during $11.2 \mathrm{ks}$ (ObsID 0201200201) and HD 161103 on 26 January 2004 during $17.6 \mathrm{ks}$ (ObsID 0201200101). In both cases, the EPIC cameras were operated in large window mode providing a time resolution of $48 \mathrm{~ms}$ and $0.9 \mathrm{~s}$ for $\mathrm{pn}$ and MOS1-2 cameras, respectively. The medium filter was used to reject optical light from the relatively bright counterparts. All data were processed with Science Analysis Software version 6.1 .0 using the most recent calibration files. Spectral analysis was performed with the Xspec-v11.3 package.

Because of the relatively high count rate of the two sources, the inclusion of times with background flares has little impact on their spectra and light curves. At $E \gtrsim 0.5 \mathrm{keV}$, spectra accumulated from the whole observation do not significantly differ from those accumulated only from low-background intervals. In the following we discard times of high background for spectral analysis (except for the Fe line complex study; Sect. 3.2.1) but do consider the whole observation for time variability studies, excluding the low-energy bands.

The resulting exposure times in the flare-free good time intervals were $9.2 \mathrm{ks}$ (MOS1 and MOS2) and $7.9 \mathrm{ks}$ (pn) for SAO 49725, and 16.6 ks (MOS1), $17.1 \mathrm{ks}$ (MOS2), and $7.6 \mathrm{ks}$ (pn) for HD 161103. In our analysis only events with pattern 0 to 4 and 0 to 12 were used for pn and MOS cameras, respectively. Source counts were extracted in circular regions, with radius of $35^{\prime \prime}$ for SAO 49725 and 40" for HD 161103 centred on the X-ray emission. The backgrounds for each observation were measured in large boxes located close to the central source and on the same CCD.

The position of the X-ray sources is fully consistent with those of the proposed optical counterparts. The angular distances of $0.7^{\prime \prime}$ and $1.8^{\prime \prime}$ between X-ray and optical positions of SAO 49725 and HD 161103, respectively, are well within the corresponding $90 \%$ confidence error radii of $1.1^{\prime \prime}$ and $3.2^{\prime \prime}$.

\section{Data analysis}

\subsection{Optical spectroscopy}

Figure 1 shows the optical spectra of SAO 49725 and HD 161103 in the $\lambda \lambda 4000-4900 \AA$ region, which is the best suited for spectral classification. The two objects have very similar spectra. A number of Fe II emission lines linked to the circumstellar environments are visible. No significant velocity change seems present during the 5-day-long ESO 3.6-m observations of HD 161103 in 2003. The radial velocities of the $\mathrm{He} \mathrm{I} \lambda 4026, \mathrm{He} \mathrm{I} \lambda 4144$, and He I $\lambda 4388$ photospheric lines of HD 161103 vary by less than $13 \mathrm{~km} \mathrm{~s}^{-1}$. For SAO 49725 , this limit is $40 \mathrm{~km} \mathrm{~s}^{-1}$ for the $\mathrm{He} \mathrm{I} \lambda 4026$ line, $17 \mathrm{~km} \mathrm{~s}^{-1}$ for the He I $\lambda 4144$, and He I $\lambda 4388$ lines on a time scale of days or years. Rotational velocities were derived using the $F W H M(\mathrm{He}$ I 24471) $-v \sin i$ correlation of Slettebak et al. (1975) (see details in Steele et al. 1999), resulting in a mean value of $v \sin i=182 \pm 33 \mathrm{~km} \mathrm{~s}^{-1}$ and $234 \pm 34 \mathrm{~km} \mathrm{~s}^{-1}$ for SAO 49725 and HD 161103, respectively. Steele et al. (1999) find a consistent value of $v \sin i=224 \pm 33 \mathrm{~km} \mathrm{~s}^{-1}$ using the He I lines in HD 161103. Also SAO 49725 and HD 161103 show very similar $\mathrm{H} \alpha$ emission lines (see Fig. 2 and Table 2), whose intensities and quasi-symmetrical profiles, both exhibiting little variability over long time intervals, suggest dense, large, and probably stable circumstellar envelopes (Slettebak et al. 1992; Tycner et al. 2005). This stability contrasts with 


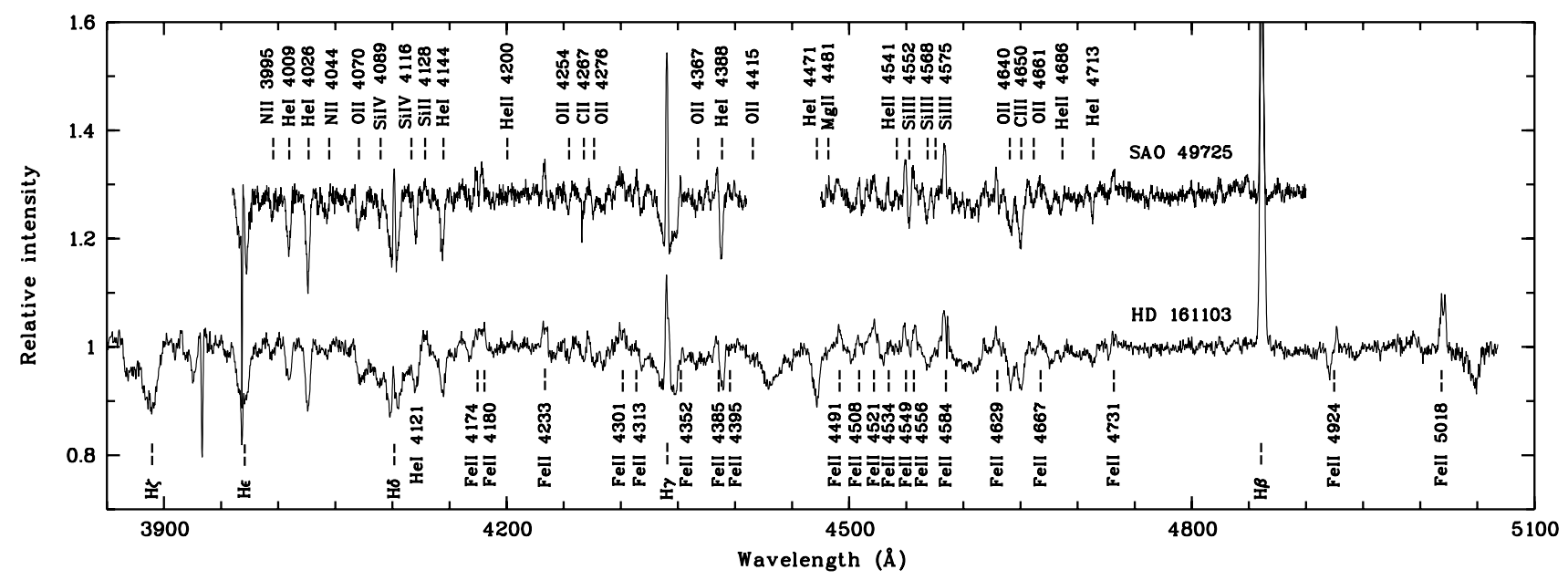

Fig. 1. The optical spectra of SAO 49725 ( $\lambda \lambda 3960-4410 \AA ̊$ on 31 July $2001 ; \lambda \lambda 4475-4900 \AA ̊$ on 1 August 2001) and HD 161103 (on 1 August 1998$)$ in the classification region. See Table 1.
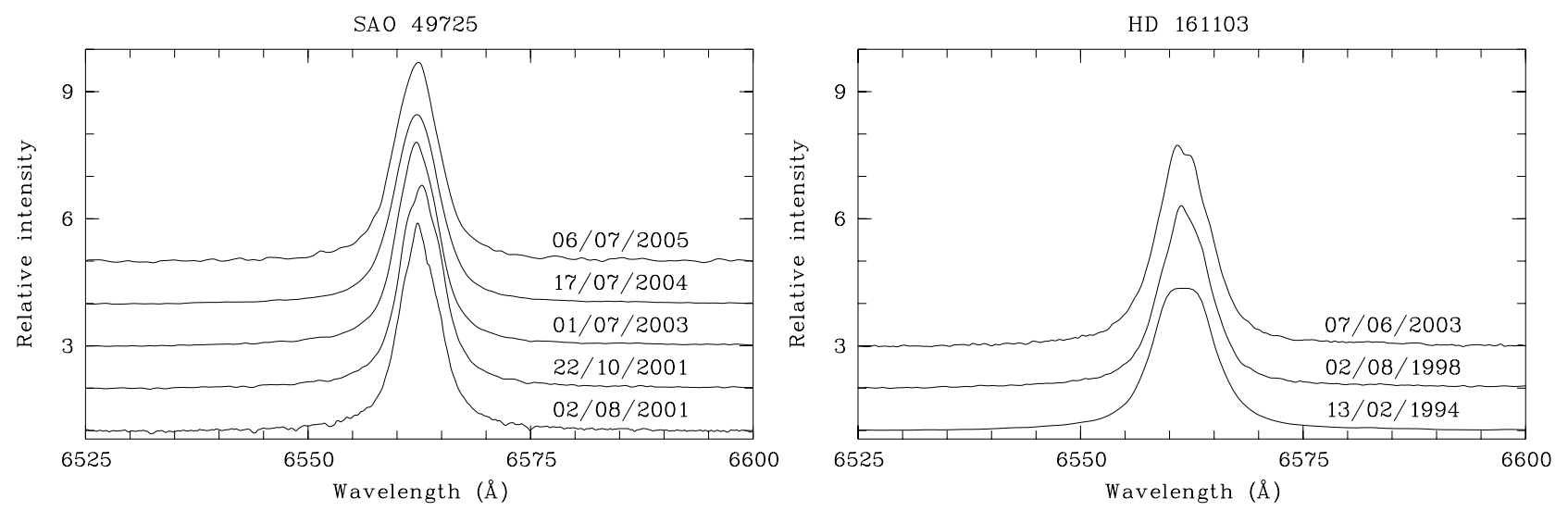

Fig. 2. The H $\alpha$ profiles of SAO 49725 and HD 161103 from observations with similar spectral resolutions. See Tables 1 and 2.

Table 2. Equivalent width of the $\mathrm{H} \alpha$ emission line in SAO 49725 and HD 161103. The errors are estimated to be $\pm 1 \AA$.

\begin{tabular}{ccc}
\hline \hline Object & Date & $\begin{array}{c}E W(\mathrm{H} \alpha) \\
(\AA)\end{array}$ \\
\hline SAO 49725 & $28 / 09 / 95$ & -30 \\
& $18 / 08 / 99$ & -32 \\
& $02 / 08 / 01$ & -30 \\
& $22 / 10 / 01$ & -32 \\
& $01 / 07 / 03$ & -31 \\
& $17 / 04 / 04$ & -32 \\
& $06 / 07 / 05$ & -30 \\
\hline HD 161103 & $13 / 02 / 94$ & -32 \\
& $02 / 08 / 98$ & -31 \\
& $07 / 06 / 03$ & -34 \\
\hline
\end{tabular}

the rather strong variability displayed by most Be/X-ray binaries on timescales of years. Among the known exceptions are A 1118-616 and Cep X-4, in which the accreting neutron stars may be in large and close to circular orbits, as suggested by Okazaki \& Negueruela (2001). The $E W(\mathrm{H} \alpha)-P_{\text {orb }}$ relation discovered by Reig et al. (1997) in Be/X-ray binaries, if applicable to these systems, would suggest $P_{\text {orb }} \gtrsim 100$ days for SAO 49725 and HD 161103. We also note that the strength of the N II $\lambda 3995$, 4044 lines may indicate that SAO 49725 and HD 161103 are moderately nitrogen-rich, as are several X-ray binary primaries.
In both stars, the Si III $\lambda 4552$ and Si IV $\lambda 4089$ lines are consistent with a B0.5 type, which also agrees with the intensity of the He II $\lambda 4686$ line seen in absorption. Later types are excluded by the weakness of the Si II $\lambda \lambda 4128-4130$ and $\mathrm{Mg}$ II $\lambda 4481$ lines. The O I triplet at $\lambda 7774(72,74$, and 75) is in emission, as is the Fe II $\lambda 7712$ line, in agreement with an early type Be star classification. The moderate emission of the metallic lines suggests a III-IV luminosity class, at least for SAO 49725, also supported by the strength of the O II $\lambda 4285$ and Si IV $\lambda 4089$ lines. Our classifications are consistent with the B0.5 III-Ve spectral type derived by Motch et al. (1997) for SAO 49725 and Steele et al. (1999) for HD 161103.

SAO 49725 and HD 161103 were also observed in the near infrared (Fig. 3). As expected for Be stars with an intense $\mathrm{H} \alpha$ line, their near-infrared spectra are dominated by the Paschen line series in emission, with positive detections up to $H_{\mathrm{P}} \lesssim 30$. The resolution in both observations was not high enough to distinguish the Ca II triplet $(\lambda 8498 / \lambda 8542 / \lambda 8662)$ from the nearby Paschen lines. The O I $\lambda 8446$ line is also seen in emission. Such feature is generally seen in Be stars to correlate well with $\mathrm{H} \alpha$ line, as it is due to excitation by $\mathrm{Ly} \beta$ photons (Andrillat et al. 1988). The Paschen and Balmer $\mathrm{H} \alpha$ lines of HD 161103 display evidence of a weak $V / R$ asymmetry.

The distance to the two stars is somewhat uncertain owing to the lack of an exact luminosity class and to the possible additional red contribution of the large circumstellar envelope 


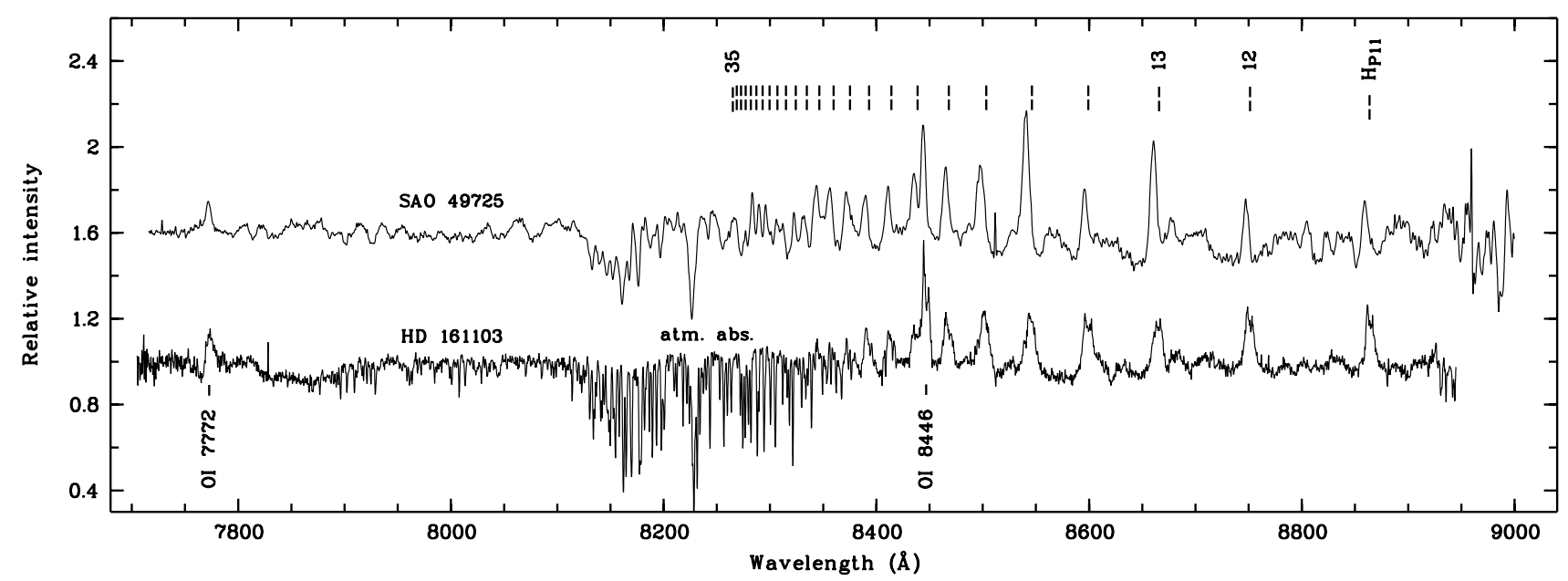

Fig. 3. The optical spectra of SAO 49725 (on 17 July 2004) and HD 161103 (on 3 August 1998) in the near infrared region. See Table 1.
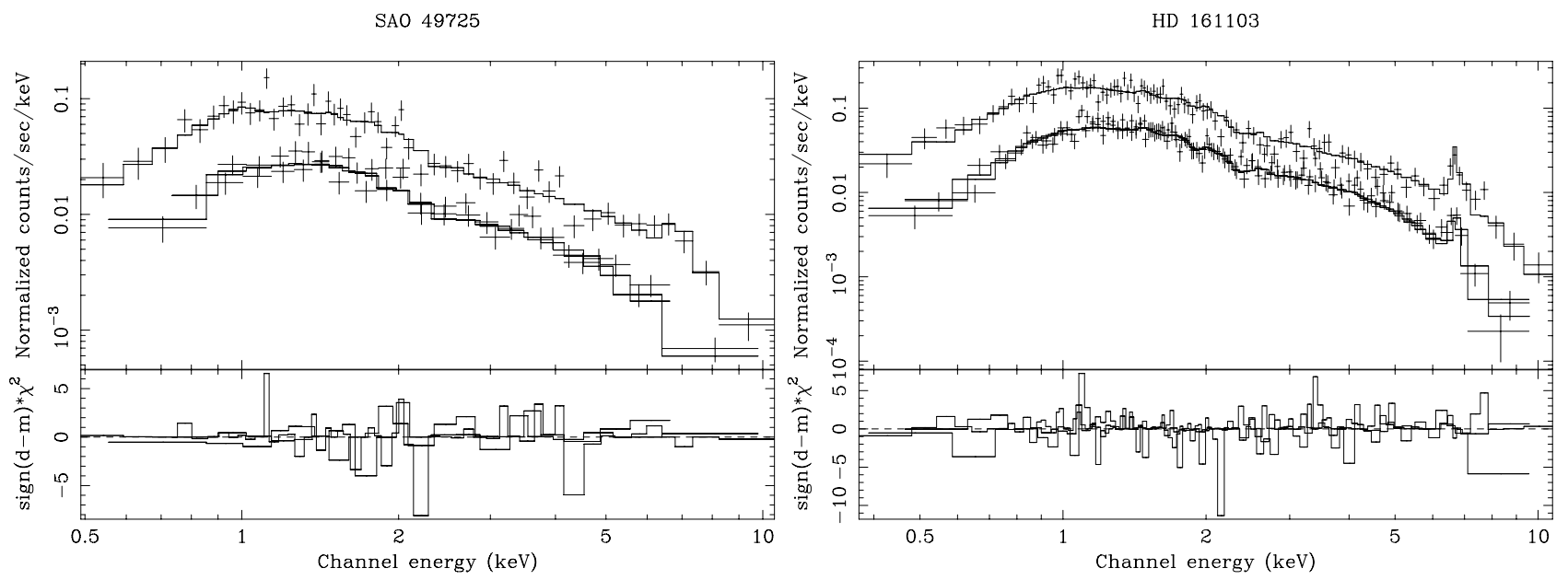

Fig. 4. EPIC spectra of SAO 49725 and HD 161103. The best-fit absorbed wabs*(mekal+mekal) model is shown for each individual EPIC camera. See Table 3.

to the intrinsic stellar colours. Assuming $V=9.23, B-V=$ 0.37 for SAO 49725, $V=8.7, B-V=0.43$ for HD 161103 (Motch et al. 1997, and references therein), and intrinsic colours and magnitudes in Wegner (1994) and Humphreys \& McElroy (1984) yields distances of 1.6 or $2.8 \mathrm{kpc}$ for SAO 49725 and 1.1 or $2.0 \mathrm{kpc}$ for HD 161103 for luminosity classes V or III, respectively. An additional conservative error of about $20 \%$ on distances probably stems from other uncertainties.

\subsection{X-ray spectroscopy}

Spectral fits were carried out simultaneously for the EPIC MOS1, MOS2, and pn cameras. Spectra were accumulated from an event list free of high background flares. In each individual spectrum, the data were grouped into energy channels containing at least 25 events.

Both SAO 49725 and HD 161103 exhibit a hard X-ray energy distribution (see Fig. 4). A strong $\mathrm{Fe} \mathrm{K} \alpha$ emission line is clearly detected in HD 161103 at $6.67 \mathrm{keV}$ with an equivalent width of $\sim 700 \mathrm{eV}$. A similar feature also seems present in SAO 49725.

Observed spectra can be equally well-fitted by an optically thin plasma (mekal) with $T \sim 10^{8} \mathrm{~K}$ and solar abundances or by a power law + iron line model with photon index $\Gamma \sim 1.5-1.8$ (see Table 3). Including a low-temperature component in these models considerably reduces the excess seen in the $\chi^{2}$-residuals around $0.9 \mathrm{keV}$ and improves the fit quality in the soft part of the spectrum. Its presence is supported by the F-test statistics with a probability of $6 \times 10^{-2}$ for SAO 49725 and $6 \times 10^{-5}$ for HD 161103 (one-mekal), 0.2 , and $5 \times 10^{-3}$ (power law + iron line) models. Freeing metal abundances does not improve the fit and yields metallicities consistent with solar. We did not find evidence of a sub-solar $\mathrm{Fe}$ abundance similar to that observed in $\gamma$ Cas (Smith et al. 2004) at the 90\% confidence level (using the vmekal model).

We also tried a model in which each mekal component is affected by a distinct absorption as for $\gamma$ Cas (Smith et al. 2004) without improving the overall fit quality. However, this configuration cannot be excluded since the absorption columns are different at the $90 \%$ confidence level. The soft component of SAO 49725 could be affected by a hydrogen column less dense than that of its hard component (Table 3). An opposite behaviour is seen in HD 161103, in which the hot component is the less absorbed. For SAO 49725 the resulting plasma temperatures are compatible with those of the model with only one absorption column. For HD 161103 the hot plasmas have the same temperatures in the two models, but the soft plasma is colder in the two-absorption model, resulting in a high emission measure and a higher luminosity by a factor $\sim 10$. We did not find acceptable 
Table 3. Best-fit parameters for the X-ray spectra of SAO 49725 and HD 161103 accumulated in time intervals free of background flares. Thermal models assume solar abundances. Quoted errors are at the $90 \%$ confidence level. Fluxes are given unabsorbed in the $0.2-12 \mathrm{keV}$ energy band and averaged over the 3 EPIC cameras. We adopted the wabs model to account for the photoelectric absorption.

\begin{tabular}{|c|c|c|c|c|c|}
\hline & \multirow[t]{2}{*}{$\overline{\text { Mekal }}$} & \multicolumn{2}{|c|}{$\overline{2-M e k a l}$} & \multirow[t]{2}{*}{ P.law + Gauss. line } & \multirow[t]{2}{*}{ Mekal + P.law + Gauss. line } \\
\hline & & 1-absorption & 2-absorptions & & \\
\hline \multicolumn{6}{|l|}{ SAO 49725 } \\
\hline$N_{\mathrm{H}_{1}}\left(10^{22} \mathrm{~cm}^{-2}\right)$ & $0.30_{-0.04}^{+0.04}$ & $0.35_{-0.06}^{+0.06}$ & $0.14_{-0.09}^{+0.11}$ & $0.35_{-0.05}^{+0.06}$ & $0.38_{-0.04}^{+0.09}$ \\
\hline$k T_{1}(\mathrm{keV})$ & $11.72_{-2.46}^{-0.046}$ & $0.87_{-0.25}^{-0.06}$ & $0.93_{-0.11}^{+0.09}$ & $\ldots$ & $0.92_{-0.25}^{+0.04}$ \\
\hline$E M_{1}{ }^{a}\left(10^{55} \mathrm{~cm}^{-3}\right)$ & $2.6(7.8)$ & $0.02(0.08)$ & $0.02(0.05)$ & $\cdots$ & $0.02(0.08)$ \\
\hline$N_{\mathrm{H}_{2}}\left(10^{22} \mathrm{~cm}^{-2}\right)$ & $\ldots$ & & $0.36_{-0.03}^{+0.05}$ & $\ldots$ & $\ldots$ \\
\hline$k T_{2}(\mathrm{keV})$ & $\ldots$ & $12.85_{-3.37}^{+7.73}$ & $12.29_{-1.48}^{+1.52}$ & $\ldots$ & $\ldots$ \\
\hline$E M_{2}{ }^{a}\left(10^{55} \mathrm{~cm}^{-3}\right)$ & $\ldots$ & $0.8(2.6)$ & $0.8(2.6)$ & $\ldots$ & $\ldots$ \\
\hline Photon index & $\ldots$ & $\ldots$ & $\ldots$ & $1.57_{-0.06}^{+0.12}$ & $1.53_{-0.10}^{+0.11}$ \\
\hline Line $(\mathrm{keV})$ & $\ldots$ & $\ldots$ & $\ldots$ & $6.61_{-0.51}^{+0.49}$ & $6.61_{-0.36}^{+0.35}$ \\
\hline$\sigma_{\text {Line }}(\mathrm{keV})$ & $\ldots$ & $\ldots$ & $\ldots$ & $\$ 1.17$ & $\$ 0.90^{-30}$ \\
\hline$f_{\mathrm{x}}\left(\mathrm{erg} \mathrm{cm}^{-2} \mathrm{~s}^{-1}\right)$ & $1.25 \times 10^{-12}$ & $1.30 \times 10^{-12}$ & $1.29 \times 10^{-12}$ & $1.38 \times 10^{-12}$ & $1.41 \times 10^{-12}$ \\
\hline$\chi_{v}^{2} /$ d.o.f. ${ }^{b}$ & $1.11 / 94$ & $1.07 / 92$ & $1.08 / 91$ & $1.16 / 91$ & $1.13 / 89$ \\
\hline \multicolumn{6}{|l|}{ HD 161103} \\
\hline$N_{\mathrm{H}_{1}}\left(10^{22} \mathrm{~cm}^{-2}\right)$ & $0.31_{-002}^{+0.02}$ & $0.35_{-0.02}^{+0.02}$ & $1.28_{-031}^{+0.24}$ & $0.38_{-003}^{+0.03}$ & $0.40_{-0.04}^{+0.05}$ \\
\hline$k T_{1}(\mathrm{keV})$ & $7.44_{-0.63}^{+1.02}$ & $0.76_{-0.17}^{+0.20}$ & $0.20_{-0.06}^{+0.10}$ & $\ldots$ & $0.80_{-0.27}^{+0.84}$ \\
\hline$E M_{1}{ }^{a}\left(10^{55} \mathrm{~cm}^{-3}\right)$ & $3.2(9.8)$ & $0.1(0.3)$ & $18.2(55.8)$ & $\ldots$ & $0.1(0.3)$ \\
\hline$N_{\mathrm{H}_{2}}\left(10^{22} \mathrm{~cm}^{-2}\right)$ & $\ldots$ & $\ldots$ & $0.36_{-0.04}^{+0.06}$ & $\ldots$ & $\ldots$ \\
\hline$k T_{2}(\mathrm{keV})$ & $\ldots$ & $8.01_{-1.00}^{+1.05}$ & $8.78_{-122}^{+1.62}$ & $\ldots$ & $\ldots$ \\
\hline$E M_{2}{ }^{a}\left(10^{55} \mathrm{~cm}^{-3}\right)$ & $\ldots$ & $1.5(9.7)$ & $3.1(9.5)$ & & \\
\hline Photon index & $\ldots$ & $\ldots$ & $\ldots$ & $1.75_{-0.06}^{+0.07}$ & $1.71_{-0.07}^{+0.07}$ \\
\hline Line $(\mathrm{keV})$ & $\ldots$ & $\ldots$ & $\ldots$ & $6.67_{-0.05}^{+0.06}$ & $6.67_{-0.05}^{+0.06}$ \\
\hline$\sigma_{\text {Line }}(\mathrm{keV})$ & $\ldots$ & $\ldots$ & $\ldots$ & $0.12_{-0.88}^{+0.08}$ & $0.12_{-0.08}^{+0.89}$ \\
\hline$f_{\mathrm{x}}\left(\mathrm{erg} \mathrm{cm}^{-2} \mathrm{~s}^{-1}\right)$ & $2.33 \times 10^{-12}$ & $2.42 \times 10^{-12}$ & $1.46 \times 10^{-11}$ & $2.79 \times 10^{-12}$ & $2.83 \times 10^{-12}$ \\
\hline$\chi_{v}^{2} /$ d.o.f. ${ }^{b}$ & $1.01 / 249$ & $0.94 / 247$ & $0.92 / 246$ & $0.97 / 246$ & $0.94 / 244$ \\
\hline
\end{tabular}

${ }^{a}$ Emission measure of the thermal component as numbered; in parenthesis assuming the largest distance (see Sect. 3.1); ${ }^{b}$ degrees of freedom.

solutions with a highly absorbed hot component similar to the one observed in $\gamma$ Cas by Smith et al. (2004).

In all cases (except for the 2-thermal model of HD 161103 with individual absorption components), the corresponding luminosities in the $0.2-12 \mathrm{keV}$ energy range are $\sim(4-12) \times$ $10^{32} \mathrm{erg} \mathrm{s}^{-1}$ depending on the assumed luminosity class and the $\mathrm{X}$-ray-to-bolometric luminosity ratio are $\sim 4 \times 10^{-6}$, almost identical for the two sources.

Interestingly, the thermal models naturally represent the $\mathrm{H}$-like and He-like iron line strengths with the correct intensity. This strongly argues in favour of a thermal interpretation of the X-ray emission of HD 161103 and SAO 49725. The corresponding emission measures (EM; Table 3 ) are dominated by the hot component and compare well with those derived for $\gamma$ Cas from RossiXTE $\left(4.1 \times 10^{55} \mathrm{~cm}^{-3}\right.$, Smith et al. 1998) and Chandra $\left(\sim 5 \times 10^{55} \mathrm{~cm}^{-3}\right.$, Smith et al. 2004).

\subsubsection{The iron $\mathrm{K} \alpha$ complex}

In order to improve the energy resolution of the EPIC pn camera, we accumulated single-pixel event spectra over the entire observations of HD 161103 and SAO 49725, i.e., including time intervals of slightly enhanced background. Spectra were grouped in bins containing a minimum of 15 events, and we used the Cashstatistic that is more appropriate for small numbers of counts.

The iron line in HD 161103 appears as a Fe K $\alpha$ complex of the neutral line at $6.4 \mathrm{keV}, \mathrm{Fe}$ XXV (6.7 keV), and Fe XXVI $(6.97 \mathrm{keV})$. Fits to the full observation time data give results consistent with those in Table 3, which were restricted to low background intervals. A hot plasma represents the emission of the $\mathrm{H}$-like and He-like lines reasonably well. A separate Gaussian line is needed to account for the Fe K fluorescent line (Fig. 5).
We estimated the contribution of each component by fitting three Gaussian lines on the power law representing the underlying continuum. The lower signal-to-noise ratio available for SAO 49725 does not allow us to resolve the Fe line complex, but the broad emission feature $(E W \sim 920 \mathrm{eV})$ is compatible with the presence of the three Fe lines detected in HD 161103. By freeing all parameters we obtain the centroid of the Gaussian lines and their estimated equivalent widths listed in Table 4. There is no evidence of non-solar abundances in any of these two sources.

These results strongly suggest that, as in $\gamma$ Cas where high resolution X-ray spectroscopy confirm it, the X-ray emission of SAO 49725 and HD 161103 is essentially due to a hot, optically thin, confined plasma, with a fluorescence feature due to reprocessing in cold matter.

\subsection{X-ray light curves}

Both X-ray light curves exhibit significant variability (see Fig. 6). The average X-ray flux of SAO 49725 slowly increases in the course of the XMM-Newton observation, while showing apparently random variations with a maximum amplitude of $\sim 80 \%$ on a time scale of a few hundred seconds. In contrast, the time behaviour of HD 161103 seems more regular during the observations. Its intensity varies almost periodically by $\sim 60 \%$ on a time scale of $\sim 3.2 \mathrm{ks}$.

\subsubsection{Spectral variations with intensity}

In order to try to disentangle different possible components contributing to the X-ray emission, we first searched for correlations between the X-ray hardness and intensity of SAO 49725 and HD 161103. We adopted the 0.5-2.0 and 2.0-12 keV 
SAO 49725

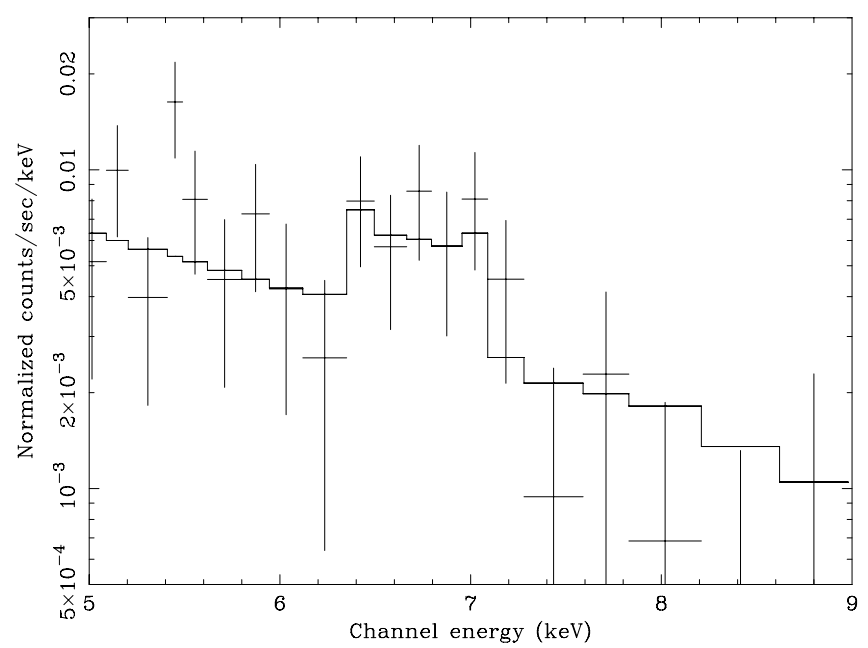

HD 161103

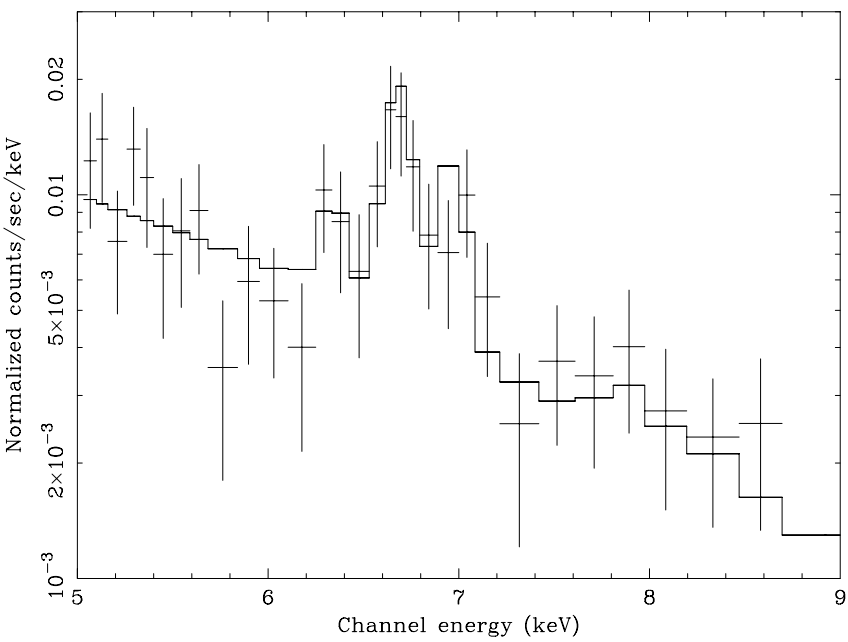

Fig. 5. The Fe line complex of HD 161103 and evidence of its existence in SAO 49725 seen in the pn single-events spectra accumulating photons in the whole observation time. The best-fit from wabs*(2-mekal + Gaussian line $)$ model are shown.

Table 4. Equivalent width of the emission iron lines in HD 161103 and SAO49725 estimated from pn single-events spectra. We use an absorbed power law model to describe the hard continuum and three Gaussians for each Fe line. Errors are given at $1 \sigma$.

\begin{tabular}{ccc}
\hline \hline Iron line $^{a}(\mathrm{keV})$ & \multicolumn{2}{c}{$E W(\mathrm{eV})$} \\
& SAO 49725 & HD 161103 \\
\hline 6.4 & $105(+145 /-84)$ & $75(+49 /-52)$ \\
6.7 & $183(+120 /-100)$ & $301(+90 /-65)$ \\
6.97 & $280(+165 /-147)$ & $133(+63 /-66)$ \\
\hline
\end{tabular}

${ }^{a}$ Frozen parameters.

energy bands to build a hardness-intensity diagram. Results for the combined EPIC cameras are shown in Fig. 7. Although the relatively small hardness ratio changes with X-ray intensity seem significant, the pattern of variability remains unclear. For HD 161103 the high state seems to correspond to the hardest energy distribution, while the opposite behaviour could be present in SAO 49725. Similar results were obtained using only EPIC pn data.

We went on to study spectral changes in HD 161103, the brightest and the most variable of the two sources, by accumulating spectra in low and high X-ray flux states for each of the three EPIC cameras. Low and high states correspond to times during which the total $0.5-12 \mathrm{keV}$ EPIC count rate is below or above of 0.53 count/s, respectively (average intensity level; see light curve in Fig. 6). As for other spectra, times of high background were discarded. We also excluded the last part of the observation $(t \gtrsim 12 \mathrm{ks})$ during which the pronounced $3.2 \mathrm{ks}$ oscillation seems to vanish. As suggested by the hardness ratio analysis, the X-ray energy distribution of HD 161103 appears slightly harder during bright X-ray states, but the best-fit parameters in the low and hard flux states are still compatible at the 90\% confidence level (see Table 5).

\subsubsection{A search for pulsations}

Timing analysis was performed using the $Z_{n}^{2}$ Rayleigh periodogram (Buccheri et al. 1983) on EPIC background corrected light curves ( $1 \mathrm{~s}, 10 \mathrm{~s}$, and $350 \mathrm{~s}$ time bins) and directly on the EPIC pn event lists. In all cases we corrected times to the barycentric system and accumulated counts in the $0.5-12 \mathrm{keV}$ energy range. The upper frequency limit is fixed by the acquisition time of $48 \mathrm{~ms}$ in the large window mode used for the pn camera $\left(f \lesssim 10.4 \mathrm{~Hz}\right.$ ). We applied the $Z_{n}^{2}$ test in $\delta f$ steps, oversampling the observation window by a factor $1000(\delta f=0.001 / T$, where $T$ is the total analysed observation time). We failed to detect high-frequency pulsations in any of the two sources, but the long period oscillation clearly visible in the light curve of HD 161103 is well detected at a period of $3245( \pm 350) \mathrm{s}$ with a pulse fraction of $24 \%$ (see Fig. 6). The upper limit on the pulse fraction of any other periodic modulation is $\sim 10 \%$ for HD 161103 and SAO 49725.

\section{Discussion}

The X-ray and optical properties of SAO 49725 and HD 161103 are very similar and can be summarised as follows:

(i) very hard X-ray spectrum, equally well fitted by a hot plasma $(k T \sim 7-12 \mathrm{keV})$ or by a power law + iron lines $(\Gamma \sim$ 1.5-1.8) model, with in both cases evidence of the presence of an additional soft thermal component $(k T \sim 0.8 \mathrm{keV})$;

(ii) presence of Fe K, He-like, and H-like iron lines (clearly detected in HD 161103, and probable in SAO 49725);

(iii) X-ray $(0.2-12 \mathrm{keV})$ luminosity of $\sim(4-12) \times 10^{32} \mathrm{erg} \mathrm{s}^{-1}$, implying an X-ray-to-bolometric luminosity ratio of $\sim 4 \times$ $10^{-6}$;

(iv) variable emission on relatively short time scales (possible stable period of $\sim 3200 \mathrm{~s}$ for HD 161103); no evidence of high-frequency pulsations;

(v) B0.5 III-Ve spectral type;

(vi) intense and quasi-symmetrical Balmer emission profile, suggesting dense and/or very large and apparently stable circumstellar envelopes.

The common properties of these two objects strikingly resemble those of $\gamma$ Cas, an enigmatic early type B0.5 IVe star that was first observed spectroscopically in 1866 (Secchi 1867). Recent Chandra observations of $\gamma$ Cas (Smith et al. 2004) show that the emission spectrum results from different plasmas: a hot-thermal dominant component $(k T \sim 11-12 \mathrm{keV})$ with low Fe abundances $(\sim 0.22 \times$ solar $)$, and two or three colder and less absorbed components $(k T \sim 0.1-3 \mathrm{keV})$ revealed by the presence 

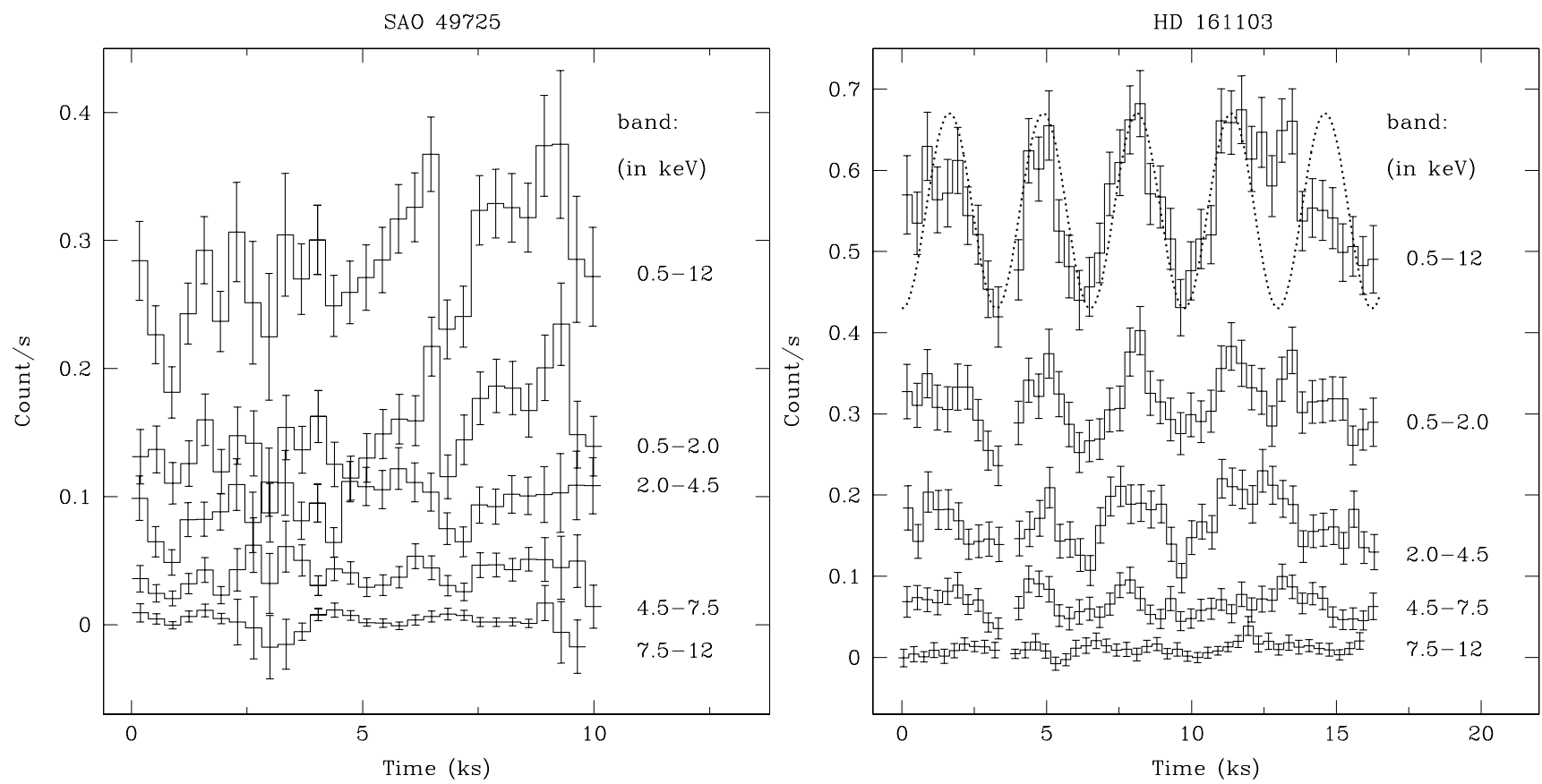

Fig. 6. EPIC (MOS1+MOS2+pn) background-subtracted barycentric corrected light curves in conventional XMM energy bands. The HD 161103 data around $t \sim 3500 \mathrm{~s}$ were lost - possibly because of a telemetric problem in the satellite. The bin size is $350 \mathrm{~s}$ in all cases. The dotted curve represents a sinusoidal modulation with period of $3245 \mathrm{~s}$ (see Sect. 3.3.2).

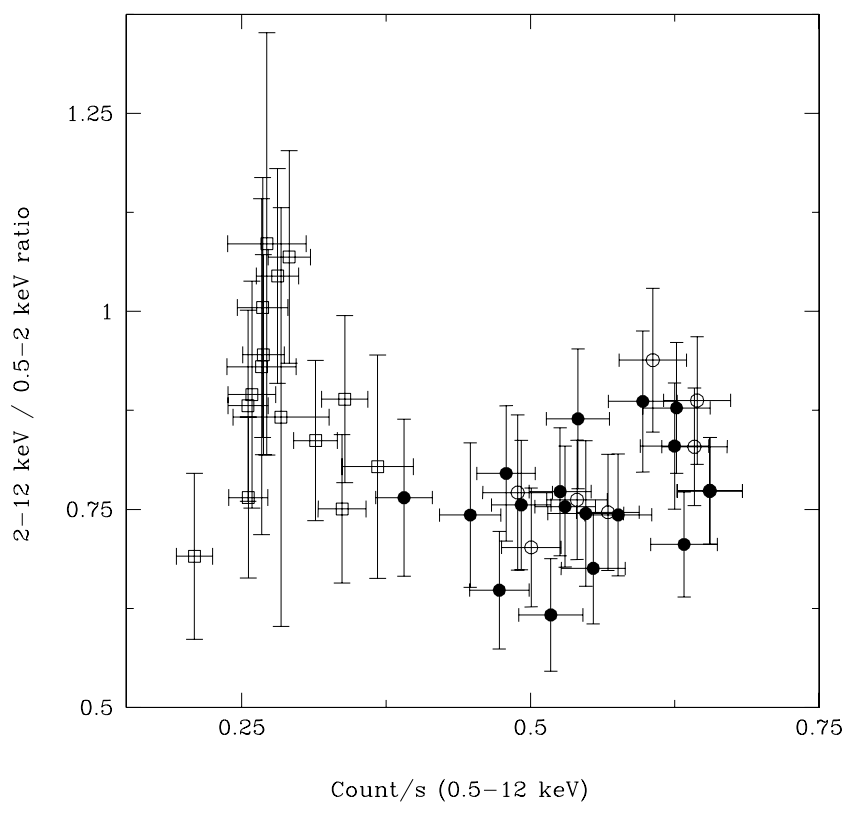

Fig. 7. Hardness-intensity diagram for SAO 49725 (open squares) and HD 161103 (filled circles for $t<12 \mathrm{ks}$, open circles for $t>12 \mathrm{ks}$ ) using all EPIC cameras simultaneously. The data were grouped in $700 \mathrm{~s}$ time bins.

of lower excitation lines from Fe L-shell and O VII-VIII. The $\mathrm{Fe} \mathrm{K} \alpha$ complex is present. The $\mathrm{X}$-ray luminosity $(2-10 \mathrm{keV})$ of $\gamma$ Cas is $\sim 5 \times 10^{32} \mathrm{erg} \mathrm{s}^{-1}$ (Kubo et al. 1998). No pulsation is detected, but there are X-ray flux variations on time scales ranging from seconds to hours (Parmar et al. 1993; Haberl 1995; Smith et al. 1998; Owens et al. 1999). In spite of several multiwavelength observing campaigns, the true nature of the source remains elusive.
Table 5. HD 161103: best-fit parameters for the low and high state $\mathrm{X}$-ray spectra. Times of high background were excluded. We also discarded the last missed oscillation ( $t>12 \mathrm{ks}$ in Fig. 6). All thermal models assume solar abundances. Quoted errors are at the $90 \%$ confidence level. Fluxes are given unabsorbed in the $0.2-12 \mathrm{keV}$ energy band.

\begin{tabular}{|c|c|c|}
\hline & \multicolumn{2}{|c|}{ State } \\
\hline & low & high \\
\hline \multicolumn{3}{|l|}{ Mekal } \\
\hline$N_{\mathrm{H}}\left(\times 10^{22} \mathrm{~cm}^{-2}\right)$ & $0.26_{-0.05}^{+0.05}$ & $0.30_{-0.03}^{+0.04}-1$ \\
\hline$k T(\mathrm{keV})$ & $7.38+38.55$ & $8.28^{+2.63}$ \\
\hline$f_{\mathrm{X}}\left(10^{-12} \mathrm{erg} \mathrm{cm}^{-2} \mathrm{~s}^{-1}\right)$ & $\sim 1.8$ & $\sim 2.6$ \\
\hline$\chi_{v}^{2} /$ d.o.f. & $1.06 / 66$ & $1.24 / 110$ \\
\hline \multicolumn{3}{|l|}{ Power law + Gaussian line } \\
\hline$N_{\mathrm{H}}\left(\times 10^{22} \mathrm{~cm}^{-2}\right)$ & $0.33_{-0.07}^{+0.08}$ & $0.34_{-0.04}^{+0.05}$ \\
\hline Photon index & $1.74+0.19$ & $1.70^{+0.12}$ \\
\hline Line (keV) & $6.67^{a}$ & $6.67^{a}$ \\
\hline$\sigma_{\text {Line }}(\mathrm{keV})$ & $0.12^{a}$ & $0.12^{a}$ \\
\hline$f_{\mathrm{X}}\left(10^{-12} \mathrm{erg} \mathrm{cm}^{-2} \mathrm{~s}^{-1}\right)$ & $\sim 2.0$ & $\sim 3.0$ \\
\hline$\chi_{v}^{2} /$ d.o.f. & $1.07 / 65$ & $1.18 / 109$ \\
\hline
\end{tabular}

${ }^{a}$ Frozen as integrated spectrum (Table 3).

Based on the similarities of $\gamma$ Cas with Be/X-ray transient pulsars in the quiescent state, the first scenario proposed was that of a compact object accreting matter from the circumstellar disc of the Be star (Marlborough et al. 1978; Murakami et al. 1986; Haberl 1995; Kubo et al. 1998). The absence of pulsations has cast some doubt on this model, although a long rotation period, a faint magnetic field, or geometrical effects could explain the lack of detection. Radial velocity variations revealed the presence of an unseen companion star in a 205 day orbit with a mass close to $1 M_{\odot}$ (Harmanec et al. 2000; Miroshnichenko et al. 2002), but it is still unclear whether the X-ray features observed in $\gamma$ Cas could be consistent with an accretion model in such an orbit (e.g. Robinson \& Smith 2000). 
Assuming that accretion on a companion star is not at work in $\gamma$ Cas, the X-ray emission could be produced by magnetic field recombination in the circumstellar disc or by fields interconnecting the star and the disc (Smith \& Robinson 1999). Dynamo effects in the upper stellar atmosphere or circumstellar disc could account for the magnetic field (Robinson et al. 2002).

We discuss the observed properties of SAO49725 and HD 161103 below in light of the single-star and accreting binary hypothesis.

\subsection{Single stars?}

The relatively modest soft X-ray luminosities radiated by SAO 49725 and HD 161103 place these objects at the boundary between early type stars and $\mathrm{Be} / \mathrm{X}$-ray transients in quiescent states, as well as persistent low-luminosity Be/X-ray systems (X Per-like). Their 0.1-2.4 keV X-ray-to-bolometric luminosity ratio is clearly above those of the vast majority of normal O-B stars as given in Berghöfer et al. (1997) (see Fig. 8). However, their much harder X-ray spectra would move them very significantly up in the $L_{\mathrm{X}} / L_{\mathrm{bol}}$ diagram if the $0.2-12 \mathrm{keV}$ band were used instead of the softer ROSAT energy range. Normal O-B stars are soft X-ray emitters with a low-temperature plasma of $\sim 0.5 \mathrm{keV}$ (Berghöfer et al. 1996), and moreover they usually display no variability on minute time scales and little on longer intervals (Meurs et al. 1992; Cohen et al. 1997; Cohen 2000). The spectral hardness, luminosity, and variability of the X-ray emission of SAO 49725 and HD 161103 are clearly incompatible with the wind-shock emission of a normal O-B star.

The most relevant single-star scenario applicable to SAO 49725 and HD 161103 is probably that proposed by Smith et al. (2004) for $\gamma$ Cas. Based on high-resolution X-ray spectroscopy from the Chandra observatory, these authors suggest a picture in which X-ray flares occur close to the surface of the Be star in magnetically confined plasmas and are partially absorbed and reprocessed in colder material located in the disc. This mechanism is supported by a pattern of correlations between optical, UV, and X-ray continuum variations and line features such as Fe and Si K fluorescence lines (Smith et al. 1998; Cranmer et al. 2000; Robinson et al. 2002; Smith et al. 2006). In $\gamma$ Cas, and also in HD 110432 (analog system; see Sect. 5), migrating sub-features are seen in the line profile of optical lines supporting the existence of corotating clouds located close to stellar surface, and probably magnetically confined (see Smith $\&$ Balona 2006), and also in agreement with the Smith et al. (2004) model. According to this model, the energy distribution seen in HD 161103 and SAO 49725 could be explained by the sum of a hard-dominant and a soft-absorbed thin thermal component, the latter representing the normal B-star shocked-wind emission.

The photoelectric absorption seen in the X-ray spectrum of SAO 49725 and HD $161103\left(N_{\mathrm{H}} \sim 3-4 \times 10^{21} \mathrm{~cm}^{-2}\right)$ is fully consistent with the one derived from the interstellar reddening of the Be stars $(E(B-V) \sim 0.6-0.7)$ estimated from optical photometry. This implies that no strong local absorption occurs.

Herbig $\mathrm{Ae} / \mathrm{Be}$ - intermediate-mass pre-main-sequence stars - were detected with X-ray luminosities of $\log L_{\mathrm{x}}$ $\left(\mathrm{erg} \mathrm{s}^{-1}\right) \sim 30-32$ and corresponding $\log L_{\mathrm{x}} / L_{\mathrm{bol}}$ from -7 to -4 (Skinner et al. 2004; Hamaguchi et al. 2005). Their X-ray spectra are usually harder than those of O-B stars and may reach thin thermal temperatures of up to $5 \mathrm{keV}$, approaching those of SAO 49725 and HD 161103 but remaining significantly cooler. They often exhibit a $\mathrm{Fe} \mathrm{K} \alpha$ line. A last similarity with the two targets studied in this paper is their significant time

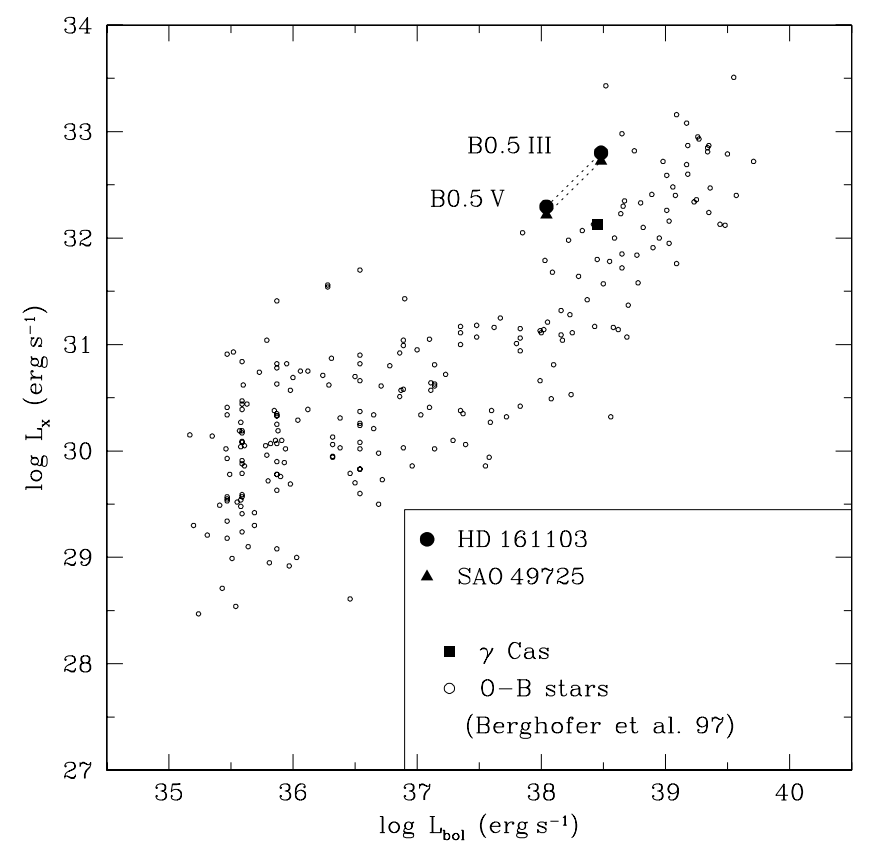

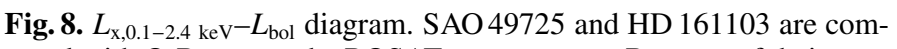
pared with O-B stars at the ROSAT energy range. Because of their very hard spectra, the X-ray luminosity excess of the new $\gamma$ Cas-like objects would be significantly larger in the $0.2-12 \mathrm{keV}$ energy band.

variability, sometimes in the form of flare-like events. There is no evidence, however, that the targets are located near starforming regions.

\subsection{Accreting binaries?}

The relatively small (typically a factor 10 in the $0.1-2.4 \mathrm{keV}$ band) excess of hard X-ray luminosity above the usual emission of normal O-B stars can be naturally explained by accretion onto a compact object. Several distinct mechanisms can account for the observed low X-ray luminosity, which is in general lower than those observed from classical Be/X-ray transients in quiescence and persistent low-luminosity Be/X-ray binaries. First, the mass accretion rate itself can be smaller than in other massive $\mathrm{X}$-ray binaries. This can happen if the local density of matter is small, i.e., if the neutron star is located outside of the circumstellar disc on a tilted orbit and is only fed by the high-velocity wind, or if the disc truncation mechanisms are extremely efficient (Okazaki \& Negueruela 2001). Alternatively, the mass accretion rate may be similar to those encountered in Be/X-ray systems, but the accretion potential well is weaker. This will be the case if the magnetized neutron star is in the propeller regime (Stella et al. 1986), where accreted matter is stopped at the magnetosphere, or if the compact object is a white dwarf.

Although the power-law indices of the energy distribution of SAO 49725 and HD 161103 compare well with those of classical Be+NS transients, in such systems the iron emission, when present, is dominated by the fluorescence line at $6.4 \mathrm{keV}$ with weak or no He-like and H-like components. The probable thermal nature of the X-ray emission of SAO 49725 and HD 161103 also disagrees with the non-thermal energy distributions always detected in accreting neutron stars. Interestingly, several cataclysmic variables with comparable X-ray luminosities display similar X-ray spectra with high $k T$ and the same iron complex in emission (see e.g. Hellier \& Mukai 2004; Pandel et al. 2005). This similarity has been considered as one of the main arguments 
for the presence of an accreting white dwarf in $\gamma$ Cas (Haberl 1995; Kubo et al. 1998).

Apart from the apparent incompatibility of the X-ray luminosity seen in $\gamma$ Cas with that expected from an accreting WD in a 205-day orbit (Robinson \& Smith 2000), one of the often invoked objections to the $\mathrm{Be}+\mathrm{WD}$ hypothesis for $\gamma \mathrm{Cas}$ is the difficulty to avoid making a neutron star in the course of a binary evolution ending with a B0.5 IV star. However, the longlasting mass-transfer phases occurring during the evolution of close binaries strongly influences the evolutionary tracks and the final stellar products. In fact, the strong dependence of the history of the system on initial conditions leads to a very wide range of configurations. Pols et al. (1991) show that Be+WD have a distribution peaking around the B2 spectral type, but are still possible for stars earlier than B0.5. In $\phi$ Per, there is a B0.5 Ve star $\left(9.3 M_{\odot}\right)$ orbited by a hot sub-dwarf with $M=$ $1.1 M_{\odot}$ (Gies et al. 1998). In the absence of further mass transfer, such a sub-dwarf will become a white dwarf and not a neutron star. There are also at least three known B+WD binaries: HR 2875 (B5 V; Motch et al. 1997; Vennes et al. 1997; Burleigh $\&$ Barstow 1998; Vennes 2000, - triple system), $\theta$ Hya (B9.5 V; Burleigh \& Barstow 1999), and 16 Dra (B9.5 V; Burleigh \& Barstow 2000). Three possible endpoints of WD + massive star systems are the binary pulsars PSR B2303+46 (van Kerkwijk \& Kulkarni 1999), PSR J1141-6545 (Kaspi et al. 2000), and PSR J1906+0746 (Lorimer et al. 2006).

\subsubsection{Accretion regimes}

The accretion regime of a neutron star, either onto its surface or its magnetosphere, depends on the relative values of the magnetospheric $\left(r_{\mathrm{m}} \propto B_{0}^{4 / 7} R_{\mathrm{NS}}^{12 / 7} M_{\mathrm{NS}}^{-1 / 7} \dot{M}_{\mathrm{acc}}^{-2 / 7}\right)$ and corotation $\left(r_{\mathrm{c}} \propto M_{\mathrm{NS}}^{1 / 3} P_{\mathrm{s}}^{2 / 3}\right)$ radius (Stella et al. 1986). Accretion on the magnetosphere $\left(r_{\mathrm{m}}>r_{\mathrm{c}}\right)$ was proposed to explain the quiescent states $\left(L_{\mathrm{X}} \sim 10^{32-36} \mathrm{erg} \mathrm{s}^{-1}\right)$ in high-mass X-ray binaries such as A 0538-66, 4U 0115+63, and V0332+53 (Corbet et al. 1997; Campana et al. 2001, 2002). Corbet et al. (1997) detected an emission iron line in A 0538-66 from ASCA observations during a quiescent state $\left(\sim 5.5 \times 10^{36} \mathrm{erg} \mathrm{s}^{-1}\right)$, which was not present during high-emission states. This feature was interpreted as a single line at $6.54 \mathrm{keV}$, or else as a blend of 6.4 and $6.7 \mathrm{keV}$ lines, and was considered as a possible signature of magnetospheric accretion.

Corbet (1984) noted that Be/X-ray binaries are located in a relatively narrow locus in the $P_{\mathrm{s}}$ versus $P_{\text {orb }}$ diagram, which could be explained as the region of spin equilibrium where $r_{\mathrm{m}} \simeq$ $r_{\mathrm{c}}$ (Waters \& van Kerkwijk 1989). In this scenario the accretion regime continuously switches between accretion onto the surface, accretion on the magnetosphere, and non-accreting states.

The accretion luminosity can be estimated by $L_{\text {acc }}(r)=$ $\eta G M \dot{M}_{\text {acc }} / r$, where $\dot{M}_{\text {acc }}$ is the accretion rate onto an object of mass $M$, in which potential-radiative energy conversion occurs with an efficiency $\eta$ at a distance $r$ from the centre of the compact object. When accretion occurs on the neutron star (white dwarf) surface, $r=R_{\mathrm{NS}}$ (or $r=R_{\mathrm{WD}}$ ). If accretion occurs on the magnetosphere $r=r_{\mathrm{m}}$, and the resulting luminosity can be estimated by:

$$
\begin{aligned}
L_{\mathrm{acc}}^{\mathrm{mag}} \simeq & 1.1 \times 10^{33}\left(\frac{M_{\mathrm{NS}}}{1.4 M_{\odot}}\right)^{8 / 7}\left[\left(\frac{B_{\mathrm{NS}, 0}}{3 \times 10^{12} \mathrm{G}}\right)\left(\frac{R_{\mathrm{NS}}}{10 \mathrm{~km}}\right)^{3}\right]^{-4 / 7} \\
& \times\left(\frac{\dot{M}_{\mathrm{acc}}}{10^{-10} M_{\odot} \mathrm{yr}^{-1}}\right)^{9 / 7} \eta \quad\left(\text { in } \mathrm{erg} \mathrm{s}^{-1}\right)
\end{aligned}
$$

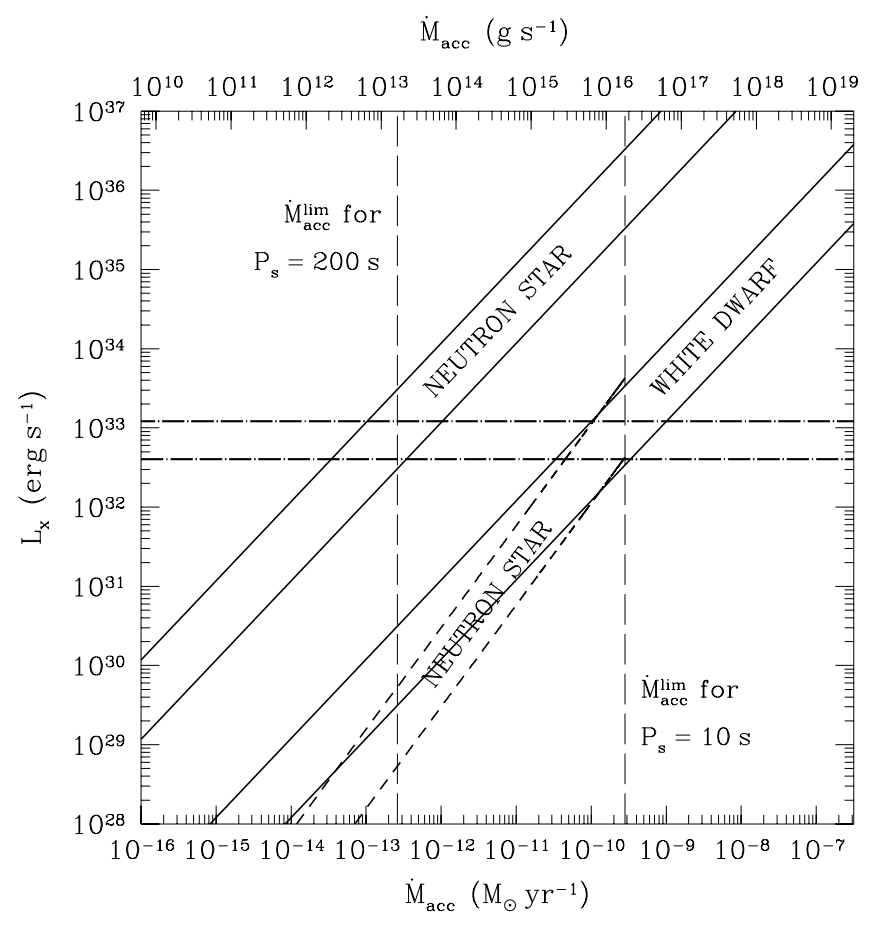

Fig. 9. Luminosity-accretion rate diagram for accretion onto the white dwarf and neutron star surfaces (solid lines, as indicated), and for magnetospheric accretion onto a neutron star (dashed lines) - upper and lower lines refer to $\eta=1$ and 0.1 , respectively. Horizontal lines show the observed X-ray luminosity (0.2-12 keV) of HD 161103 and SAO 49725 . Vertical lines mark $\dot{M}_{\text {acc }}^{\lim }$ for a neutron star with $P_{\mathrm{s}}=$ 10 and $200 \mathrm{~s}$ (see text).

The transition between accretion on the magnetosphere and accretion on the neutron star ideally occurs at an accretion rate $\left(\dot{M}_{\mathrm{acc}}^{\mathrm{lim}}\right)$ for which $r_{\mathrm{m}}=r_{\mathrm{c}}$ and should be accompanied by a several orders of magnitude jump in X-ray luminosity (Corbet 1996).

Figure 9 shows $L_{\text {acc }}$ versus $\dot{M}_{\text {acc }}$ for three cases: accretion onto the neutron star surface, accretion onto the neutron star magnetosphere, and accretion onto a weakly magnetized white dwarf. We adopt a bipolar description for the magnetic field of the neutron star with $B_{\mathrm{NS}, 0}=3 \times 10^{12} \mathrm{G}$ at the pole; masses and radii of $1.4 M_{\odot}$ and $10 \mathrm{~km}$ for the neutron star and $1.0 M_{\odot}$ and $0.01 R_{\odot}$ for the white dwarf. We assume accretion efficiencies $\eta$ of 1.0 and 0.1 .

The observed X-ray luminosities of HD 161103 and SAO $49725\left(\sim(4-12) \times 10^{32} \mathrm{erg} \mathrm{s}^{-1}\right.$ at $\left.0.2-12 \mathrm{keV}\right)$ could thus be produced by direct accretion on a neutron star surface for an accretion rate of $\sim 2 \times 10^{-13} M_{\odot} \mathrm{yr}^{-1}$ corresponding to $r_{\mathrm{m}} \sim$ $6 \times 10^{9} \mathrm{~cm}$. This requires the opening of the magnetic barrier, i.e., neutron star rotation periods larger than $\sim 200 \mathrm{~s}$. The same X-ray luminosity could be explained by accretion on the magnetosphere of a neutron star with a spin period shorter than $\sim 10 \mathrm{~s}$ corresponding to $r_{\mathrm{m}} \sim 8 \times 10^{8} \mathrm{~cm}$ or onto a white dwarf assuming a much higher accretion rate of $\sim 2 \times 10^{-10} M_{\odot} \mathrm{yr}^{-1}$.

\subsubsection{The likelihood of the accretion scenario}

Estimating X-ray luminosities consistent with the various accretion regimes requires a good understanding of the density and velocity fields of the circumstellar matter along the orbit of the accreting star. 
A growing number of observational evidences and theoretical developments have reinforced the idea that the viscous decretion disc model proposed by Lee et al. (1991) can explain most of the disc-related observational properties of Be stars. A major step forward was the recognition that radial outflow velocities were highly subsonic in the whole line-emitting disc region. Tangential velocities are mainly Keplerian, and the viscosity acts in an opposite way as in an accretion disc, transporting the angular momentum of the inner regions toward the outer disc. This scenario has been successful in explaining the observed Balmer line profiles (Hanuschik 2000) and the IR colours (Porter 1999). Negueruela \& Okazaki (2001) have shown that the tidal torque of a stellar companion can efficiently truncate the viscous disc at a resonance radius that depends on the disc viscosity, on the mass ratio of the stars, and on the orbital parameters. Okazaki \& Negueruela (2001) have applied this model to the class of Be/X-ray transients and shown that many of the X-ray and optical behaviour of these transients (e.g. $V / R$ variations, occurrence of type I or II outbursts) could be explained in this framework. Disc truncation should be more efficient in systems with small orbital eccentricities and small orbital periods (Zhang et al. 2004) and could deplete the circumstellar disc at relatively short distances from the Be star.

Unfortunately, the decretion disc model makes no prediction for the density and velocity fields beyond the truncation radius. Even in the absence of truncation, the density and velocity laws at the outer edge of the disc, beyond the trans-sonic point are illdefined. At a large distance from the star, it is reasonable to think that the pressure of the high-velocity stellar wind will considerably change the flaring disc structure (Kubat 2006) dragging the disc material to higher velocities.

Direct accretion on a neutron star implies rather extreme conditions. First, the spin period must have slowed down to large values, although not unusual ones, in order for accretion to take place. Second, the orbital period must be long enough to explain the low accretion rate. Persistent Be/X-ray sources with low luminosity, such as X Per $\left(P_{\text {orb }}=250^{\mathrm{d}}\right)$ or RX J0146.9+6121, with $X$-ray luminosities of the order of 10 to 100 times what is observed from SAO 49725 or HD 161103 usually do not display any iron line (Mereghetti et al. 2000; Coburn et al. 2001). The absence of a fluorescence iron line probably reflects the scarcity of the circumstellar medium at the large distances where the accreting neutron star orbits. In this respect, the strength of the iron line in HD 161103 and SAO 49725 suggests instead that the $\mathrm{X}$-ray source is embedded in a relatively dense cold medium, casting severe doubt on the accreting neutron star hypothesis.

Accretion onto a white dwarf (or onto the magnetosphere of a fast spinning neutron star) requires much higher accretion rates. If the X-ray luminosity scales with the gravitational potential well we expect the hypothetical white dwarf to be on the same kind of orbit than neutron stars with X-ray luminosities of the order of $10^{35-36} \mathrm{erg} \mathrm{s}^{-1}$, comparable to those of some quiescent states of A $0535+26$, for instance (Motch et al. 1991). A 0535+26 displays a rich behaviour with regular type I outbursts occurring close to periastron passage, rare type II outbursts, and it undergoes long periods of low X-ray luminosity during which no outburst occurs at periastron. Disc truncation can explain these contrasted variability regimes adequately (Okazaki \& Negueruela 2001). Because of the absence of birth kick velocity, white dwarf eccentricities should be significantly smaller than those of neutron stars and, as a consequence, disc truncation efficiency should be much higher, leading to smaller discs. Replacing the neutron star in A 0535+26 in a non-outbursting state by a white dwarf could perhaps explain the mean X-ray luminosity and the apparent absence of type I or II outbursts in $\gamma$ Cas like objects. In general, an orbiting white dwarf may truncate the Be disc so efficiently that it will only accrete from matter escaping beyond the truncation radius, in agreement with the low X-ray luminosities and the apparent absence of outbursts. However, this scenario has to face a major difficulty, namely that optical observations show that the circumstellar discs in $\gamma$ Cas and also probably in its twins are large and stable in contradiction to what is expected from truncated discs.

\subsubsection{Be + black hole systems?}

Black hole transients in quiescent states (BHQ) have power-law spectra with photon index ranging between 0.9 and 2.3 (mean index of $\sim 1.5)$ and luminosity $(0.3-7 \mathrm{keV})$ of $\sim 10^{30-33} \mathrm{erg} \mathrm{s}^{-1}$ (Garcia et al. 2001; Kong et al. 2002; Hameury et al. 2003). These power-law indices are in principle similar to those derived for SAO 49725 and HD 161103, although a Fe K $\alpha$ complex is not seen in BHQ. The black hole hypothesis is ruled out by the orbital solution proposed for $\gamma$ Cas (Harmanec et al. 2000) but may still work for our targets. As suggested by Zhang et al. (2004) the effective truncation of the decretion disc around the Be star implies long quiescent phases of low X-ray luminosity, if the accreting black hole is in a narrow orbit ( $\$ 30$ days) of low eccentricity. But the stability of the circumstellar disc might not be consistent with this scenario.

\subsection{What is the nature of the 3200 s oscillation in HD 161103?}

The short duration of the X-ray observation does not allow us to constrain the stability of the $3200 \mathrm{~s}$ period detected in the X-ray light curve of HD 161103. Such a long period can be due to a spinning white dwarf or neutron star, and its confirmation would be a major step forward in understanding these systems. In the case of $2 \mathrm{~S} 0114+650$, a $\sim 2.78$-h oscillation is detected, which probably reflects the spin period of a neutron star emitting an X-ray luminosity of $L_{\mathrm{X}} \sim 10^{35} \mathrm{erg} \mathrm{s}^{-1}(2-10 \mathrm{keV}$; Finley et al. 1992).

A similar quasi-periodic time behaviour is often observed in $\gamma$ Cas (Parmar et al. 1993; Haberl 1995; Smith et al. 1998; Owens et al. 1999) with comparable time scales. This variability has been interpreted in terms of flare-like events similar to those detected from the hot coronae of solar-like stars (Smith et al. 2004). This quasi-periodic flaring behaviour also compares well with that of the Be/X-ray binary EXO 2030+375 (Parmar et al. 1989), in which a series of six X-ray flares with duration between 80 and 130 min was observed with recurrence times of $\sim 230 \mathrm{~min}$. A last possible analogy is the flaring activity on timescales of $\sim 1 \mathrm{~h}$ detected in $4 \mathrm{U} 2206+54$ by Masetti et al. (2004).

Although $\beta$ Cephei stars exhibit optical photometric pulsations with comparable periods of the order of 3-6 h, they usually display little X-ray variability (Cohen 2000; Cassinelli et al. 1994) and are thus unlikely to be related to the phenomenon we observed.

\section{A new class of X-ray emitters}

Currently four Be stars with X-ray and optical properties that are very similar to $\gamma$ Cas, HD 161103, and SAO 49725 have been serendipitously discovered in the XMM-Newton surveys (see Motch et al. 2006a, for a recent review). Examples are 
USNO 0750-13549725 and SS 397 (Motch et al. 2003, 2006a,b). The first object is a blue straggler B1 IIIe star at the centre of NGC 6649 (Marco et al. 2006) - an open cluster $\sim 1.6 \mathrm{kpc}$ distant and heavily reddened $\left(A_{\mathrm{V}} \sim 4.95\right)$, with an estimated age of 50 Myr (Turner 1981; Walker \& Laney 1987). Its X-ray spectrum can be fitted by a power law model with $\Gamma \sim 1.4-1.6$ or by a mekal model with $k T \sim 10 \mathrm{keV}$. The resulting X-ray $(0.2-12 \mathrm{keV})$ luminosity is $\sim 3 \times 10^{32} \mathrm{erg} \mathrm{s}^{-1}$.

The second object, SS 397, is a B0.5 Ve star and also a hard $\mathrm{X}$-ray emitter. The temperature, as inferred by a mekal model fit, is $k T \sim 13 \mathrm{keV}$, or $\Gamma \sim 1.6-1.8$ from the power law model. An emission iron line, or blend of the $\mathrm{Fe} \mathrm{K} \alpha$ complex, was also observed. Strong flux variations in both USNO 0750-13549725 and SS 397 were detected by XMM-Newton observations, on very similar time scales as found here in HD 161103 and SAO 49725 (Sect. 3.3). Detailed results on these new sources will be presented elsewhere. A BeppoSAX observation of the HEAO-1 Be/X-ray binary candidate HD 110432 (B0.5 IIIe) was reported by Torrejón \& Orr (2001). Its X-ray spectrum was also described well by a thermal hot plasma of $k T \sim 11 \mathrm{keV}-$ resulting in an unabsorbed luminosity $(2-10 \mathrm{keV})$ of $\sim 3.4-7 \times$ $10^{32} \mathrm{erg} \mathrm{s}^{-1}$. A possible periodicity of $\sim 14 \mathrm{ks}$ was suspected. As in $\gamma$ Cas, optical spectroscopy of HD 110432 revealed migrating sub-features in the line profiles suggesting the existence of magnetically confined circumstellar clouds corotating close to the star's surface (see results and discussion in Smith \& Balona 2006).

It is now clear that the long-standing $\gamma$ Cas puzzle is no longer an unique case, as several new $\gamma$ Cas analogs have been found in X-ray surveys. Amazingly, their X-ray and optical properties fall in a very narrow range, in particular their spectral types which are all consistent with $\sim \mathrm{B} 0.5 \mathrm{e}$. They constitute $a$ new and well-defined class of $X$-ray emitters that is characterised by: [1] a hard X-ray emission that is very likely thermal and dominated by a component with $7 \lesssim k T(\mathrm{keV}) \lesssim 13$ as supported by the presence of a prominent $\mathrm{Fe} \mathrm{K} \alpha$ complex, [2] a variable behaviour, [3] a moderate $0.2-12 \mathrm{keV}$ luminosity $\left(32 \lesssim \log L_{\mathrm{x}}\left(\mathrm{erg} \mathrm{s}^{-1}\right) \lesssim 33\right)$, and from their optical properties, by [4] a large and probably stable circumstellar disc.

\section{Conclusions}

Our XMM-Newton observations of two low-luminosity hard $\mathrm{X}$-ray emitting Be stars selected from the ROSAT all-sky survey reveal a very likely thermal emission of high temperature $(k T \gtrsim 8 \mathrm{keV})$. A strong iron line, including a fluorescence component, was detected. They also share very similar optical properties such as spectral types (B0.5 III-Ve) and strength of the Balmer emission $(E W \sim-31 \AA)$. This pattern of common X-ray and optical properties appears quite similar to that of the so far unique star $\gamma$ Cas and points to the emergence of a new class of $\gamma$ Cas analogs.

HD 161103 displays a $3200 \mathrm{~s}$ oscillation that could be the signature of a rotating accreting compact object or, with equal likelihood, the analog of the long period quasi-oscillations seen in the X-ray observations of $\gamma$ Cas. We discuss the possible X-ray emission mechanisms in the framework of the models proposed for $\gamma$ Cas. A single-star scenario in which X-ray flares occur close to the stellar photosphere in magnetically confined regions should be able to account for the observations but may be challenged by the low intrinsic absorption of the X-ray source. This model can neither be really proved nor falsified with the presently available data. The scenario with accretion onto a neutron star or a white dwarf faces a number of difficulties, one of the most severe being the absence of evidence for disc truncation effects. The strength of the $6.4 \mathrm{keV}$ line strongly argues against accretion onto a neutron star on a long-period orbit.

Acknowledgements. We would like to thank Dr. Myron A. Smith for useful discussions. The INT is operated on the island of La Palma by the Isaac Newton Group in the Spanish Observatorio del Roque de Los Muchachos of the Instituto de Astrofísica de Canarias. The G. D. Cassini telescope is operated at the Loiano Observatory by the Osservatorio Astronomico di Bologna. Based in part on observations made at Observatoire de Haute Provence (CNRS), France. Partially based on data collected at the European Southern Observatory, Chile (71.D0151). R.L.O. acknowledges financial support from Brazilian agencies FAPESP (grant 03/06861-6) and CAPES (grant BEX0784/04-4), and the Observatoire de Strasbourg. I.N. is a researcher of the programme Ramón y Cajal, funded by the Spanish Ministerio de Ciencia y Tecnología (currently Ministerio de Educación y Ciencia) and the University of Alicante, with partial support from the Generalitat Valenciana and the European Regional Development Fund (ERDF/FEDER). This research is partially supported by the MCyT (currently MEC) under grant AYA2002-00814.

\section{References}

Andrillat, Y., Jaschek, M., \& Jaschek, C. 1988, A\&AS, 72, 129

Berghöfer, T. W., Schmitt, J. H. M. M., \& Cassinelli, J. P. 1996, A\&AS, 118, 481 Berghöfer, T. W., Schmitt, J. H. M. M., Danner, R., \& Cassinelli, J. P. 1997, A\&A, 322, 167

Buccheri, R., Bennett, K., Bignami, G. F., et al. 1983, A\&A, 128, 245

Burleigh, M. R., \& Barstow, M. A. 1998, MNRAS, 295, L15

Burleigh, M. R., \& Barstow, M. A. 1999, A\&A, 341, 795

Burleigh, M. R., \& Barstow, M. A. 2000, A\&A, 359, 977

Campana, S., Gastaldello, F., Stella, L., et al. 2001, ApJ, 561, 924

Campana, S., Stella, L., Israel, G. L., et al. 2002, ApJ, 580, 389

Cassinelli, J. P., Cohen, D. H., Macfarlane, J. J., Sanders, W. T., \& Welsh, B. Y. 1994, ApJ, 421, 705

Coburn, W., Heindl, W. A., Gruber, D. E., et al. 2001, ApJ, 552, 738

Cohen, D. H. 2000, in The Be Phenomenon in Early-Type Stars, ed. M. Smith,

H. F. Henrichs, \& J. Fabregat (San Francisco: ASP), IAU Colloq., 175, 156

Cohen, D. H., Cassinelli, J. P., \& MacFarlane, J. J. 1997, ApJ, 487, 867

Corbet, R. H. D. 1984, A\&A, 141, 91

Corbet, R. H. D. 1996, ApJ, 457, L31

Corbet, R. H. D., Charles, P. A., Southwell, K. A., \& Smale, A. P. 1997, ApJ, 476,833

Cranmer, S. R., Smith, M. A., \& Robinson, R. D. 2000, ApJ, 537, 433

Finley, J. P., Belloni, T., \& Cassinelli, J. P. 1992, A\&A, 262, L25

Garcia, M. R., McClintock, J. E., Narayan, R., et al. 2001, ApJ, 553, L47

Gies, D. R., Bagnuolo, W. G., Ferrara, E. C., et al. 1998, ApJ, 493, 440

Haberl, F. 1995, A\&A, 296, 685

Hamaguchi, K., Yamauchi, S., \& Koyama, K. 2005, ApJ, 618, 360

Hameury, J.-M., Barret, D., Lasota, J.-P., et al. 2003, A\&A, 399, 631

Hanuschik, R. W. 2000, in The Be Phenomenon in Early-Type Stars, ed. M.

Smith, H. F. Henrichs, \& J. Fabregat (San Francisco: ASP), IAU Colloq., 175,518

Harmanec, P., Habuda, P., Stefl, S., et al. 2000, A\&A, 364, L85

Hellier, C., \& Mukai, K. 2004, MNRAS, 352, 1037

Humphreys, R. M., \& McElroy, D. B. 1984, ApJ, 284, 565

Kaspi, V. M., Lyne, A.G., Manchester, R. N., et al. 2000, ApJ, 543, 321

Kong, A. K. H., McClintock, J. E., Garcia, M. R., Murray, S. S., \& Barret, D. 2002, ApJ, 570, 277

Kubat, J. 2006, in proceedings of the conference on Active O-B stars, Sapporo, Sept. 2005, ed. S. Stefl, S. Owocki, \& A. Okazaki, ASP Conf. Ser.

Kubo, S., Murakami, T., Ishida, M., \& Corbet, R. H. D. 1998, PASJ, 50, 417

Lee, U., Saio, H., \& Osaki, Y. 1991, MNRAS, 250, 432

Lorimer, D. R., Stairs, I. H., Freire, P. C. C., et al. 2006, ApJ, 640, 428

Lucy, L. B., \& Solomon, P. M. 1970, ApJ, 159, 879

Marco, A., Negueruela, I., \& Motch, C. 2006, in Proceedings of Massive Stars in Interacting Binaries, Hotel Sacacomie, Montreal, Quebec, Canada, August 2004, ed. A. F. J. Moffat, \& N. St.-Louis, ASP Conf. Ser., in press Marlborough, J. M., Snow, T. P., \& Slettebak, A. 1978, ApJ, 224, 157 Masetti, N., Dal Fiume, D., Amati, L., et al. 2004, A\&A, 423, 311 Mereghetti, S., Tiengo, A., Israel, G. L., \& Stella, L. 2000, A\&A, 354, 567 Meurs, E. J. A., Piters, A. J. M., Pols, O. R., et al. 1992, A\&A, 265, L41 Miroshnichenko, A. S., Bjorkman, K. S., \& Krugov, V. D. 2002, PASP, 114, 1226 Motch, C., Stella, L., Janot-Pacheco, E., \& Mouchet, M. 1991, ApJ, 369, 490 
Motch, C., Haberl, F., Dennerl, K., Pakull, M., \& Janot-Pacheco, E. 1997, A\&A 323,853

Motch, C., Herent, O., \& Guillout, P. 2003, Astron. Nachr., 324, 61

Motch, C., Lopes de Oliveira, R., Negueruela, I., Haberl, F., \& Janot-Pacheco, E. 2006a, in proceedings of the conference on Active O-B stars, Sapporo, Sept. 2005, ed. S. Stefl, S. Owocki, \& A. Okazaki, ASP Conf. Ser.

Motch, C., Barcons, X., Carrera, F., et al. 2006b, A\&A, submitted

Murakami, T., Koyama, K., Inoue, H., \& Agrawal, P. C. 1986, ApJ, 310, L31

Negueruela, I., \& Okazaki, A. T. 2001, A\&A, 369, 108

Okazaki, A. T., \& Negueruela, I. 2001, A\&A, 377, 161

Owens, A., Oosterbroek, T., Parmar, A. N., et al. 1999, A\&A, 348, 170

Pandel, D., Córdova, F. A., Mason, K. O., \& Priedhorsky, W. C. 2005, ApJ, 626, 396

Parmar, A. N., White, N. E., Stella, L., Izzo, C., \& Ferri, P. 1989, ApJ, 338, 359

Parmar, A. N., Israel, G. L., Stella, L., \& White, N. E. 1993, A\&A, 275, 227

Pols, O. R., Coté, J., Waters, L. B. F. M., \& Heise, J. 1991, A\&A, 241, 419

Porter, J. M. 1999, A\&A, 348, 512

Raguzova, N. V. 2001, A\&A, 367, 848

Raguzova, N. V., \& Popov, S. B. 2005, Astron. Astrophys. Trans, 24, 151

Reig, P., Fabregat, J., \& Coe, M. J. 1997, A\&A, 322, 193

Robinson, R. D., \& Smith, M. A. 2000, ApJ, 540, 474

Robinson, R. D., Smith, M. A., \& Henry, G. W. 2002, ApJ, 575, 435

Secchi, A. 1867, Astron. Nachr., 68, 63

Skinner, S. L., Güdel, M., Audard, M., \& Smith, K. 2004, ApJ, 614, 221

Slettebak, A., Collins, G. W., Boyce, P. B., White, N. M., \& Parkinson, T. D. 1975, ApJS, 281, 137
Slettebak, A., Collins, G. W., \& Truax, R. 1992, ApJS, 81, 335

Smith, M. A., \& Robinson, R. D. 1999, ApJ, 517, 866

Smith, M. A., \& Balona, L. 2006, ApJ, 640, 491

Smith, M. A., Robinson, R. D., \& Corbet, R. H. 1998, ApJ, 503, 877

Smith, M. A., Cohen, D. H., Gu, M. F., et al. 2004, ApJ, 600, 972

Smith, M. A., Henry, G. W., \& Vishniac, E. 2006, ApJ, submitted, personal communication

Steele, I. A., Negueruela, I., \& Clark, J. S. 1999, A\&AS, 137, 147

Stella, L., White, N. E., \& Rosner, R. 1986, ApJ, 308, 669

Tycner, C., Lester, J. B., Hajian, A. R., et al. 2005, ApJ, 624, 359

Torrejón, J. M., \& Orr, A. 2001, A\&A, 377, 148

Turner, D. G. 1981, AJ, 86, 231

van Bever, J., \& Vanbeveren, D. 1997, A\&A, 322, 116

van den Heuvel, E. P. J., \& Rappaport, S. A. 1987, in Physics of Be Stars, ed. A.

Slettebak, \& T. P. Snow (Cambridge University Press), IAU Colloq., 92, 291 van Kerkwijk, M. H., \& Kulkarni, S. R. 1999, ApJ, 516, L25

Vennes, S. 2000, A\&A, 354, 995

Vennes, S., Berghofer, T. W., \& Christian, D. J. 1997, ApJ, 491, L85

Zhang, F., Li, X.-D., \& Wang, Z.-R. 2004, ApJ, 603, 663

Walker, A. R., \& Laney, C. D. 1987, MNRAS, 224, 61

Waters, L. B. F. M., \& van Kerkwijk, M. H. 1989, A\&A, 223, 196

Waters, L. B. F. M., Pols, O. R., Hogeveen, S. J., Coté, J., \& van den Heuvel, E. P. J. 1989, A\&A, 220, L1

Wegner, W. 1994, MNRAS, 270, 229 